\title{
Use of electronic mail for patient record transmission
}

\author{
JOHN F COWIE
}

\section{Introduction}

The increasing use of computers for patient data and for word processing has led to an upsurge of interest in the use of electronic mail for transmission of patient records. The requirement of the 1984 Data Protection Act that all reasonable precautions should be taken to prevent such records being exposed to unauthorised inspection has, however, seemed an overwhelming obstacle. This article describes a method that is being used successfully for transmitting laboratory data between two hospital departments. The scheme is intended to form the basis of a larger pilot study to link various hospitals and departments in the Lothian Health Board area. It uses encryption/decryption programs, with error correction routines, to ensure complete data protection during transmission. The programs are adaptable to any computer that has disc drives. Telecom Gold is the electronic mail service used.

The principle of electronic mail is simple. Using a modem (a device that enables the connection of a computer to a telephone) and a telephone link, text from one computer can be transferred to a reserved area of memory on a host computer. This area of reserved memory is rented by a user and is known as his postbox. The user can obtain access to his mail by calling up the host computer and typing his password. The text is then copied to the user's computer over the telephone line. Although the host computer may be hundreds of miles away, access to data in most parts of the country is possible using only a local telephone call as in many major towns British Telecom has set up special numbers linked to a dedicated device that transfers data between computers. The total cost of sending mail electronically compares favourably with the cost of using normal mail and it is much quicker; a letter can even be sent from a postbox in Britain to a postbox in Australia in just a few minutes.

This system has many attractions:

(1) Use of local exchanges minimises interference due to poor telephone lines.

(2) The data can be sent whenever it is convenient for the sender, and the data can be retrieved at a time convenient for the user.

(3) Speedy transmission with nominal 24 hour, seven day a week availability (actual availability exceeds $99 \%$ ).

(4) There is no two way access either to the sending or to the receiving computer, so there is no possibility of illegal access to all patients' records. The only point that is vulnerable to attack is the postbox on the host computer. Although that limits the amount of information at risk, it is still unacceptable under the terms of the Data Protection Act.

There are, however, still more points to be considered before such a system could be used for patient data, especially laboratory data.

\section{Method}

It was decided that the following specification had to be met before any system could be approved, even on a trial basis:

(1) Completely faithful transmission (characters must not be altered or dropped).

(2) High reliability (retransmission requests must be a rare event).

(3) High security (no unauthorised access).

From preliminary experience it seems that the rate of faulty character

Medical Renal Unit, Royal Infirmary of Edinburgh, Edinburgh EH3 9YW JOHN F COWIE, BSC, biochemist transmission with our equipment and telephone lines is about 1 in 4000 characters; this is certainly too high for direct data transmission.

This problem could be overcome by using RAP software-error correcting software for use on electronic mail-but this has the twin disadvantages of being relatively expensive and of running on a limited and expensive range of microcomputers. Using this technique, however, would take care of the first two requirements, but what of the third-no unauthorised access?

The password requirements for each electronic postbox will certainly keep out most would be browsers, but it has been shown publicly that these may not be enough to keep out the new breed of electronic vandal.

Although at present the possibility of that happening to our files is small, the likely expansion of electronic mail, the increasing numbers of computer literate youths (who now have books and games available to teach them how to break into the networks), and, above all, the extreme sensitivity of patient data, mean that such data must be fully protected. Encryption can give that protection.

It was decided to write a program that combined a reasonable measure of error correction with a secure method of encryption and that could be used on any computer with disc drives. The proviso that it should be usable on any computer is necessary because organisations such as health authorities or boards have almost every possible type of microcomputer in use.

The advantage of RAP software is that it actually checks the data as these are sent to, or received from, the host computer. If a faulty character is received it is rejected and retransmission occurs until the correct character is received. Normal transmission-for example, without RAP software-does no error checking during transmission so any error checking must take place after the message has been received.

To understand our method it is necessary to know that text is transmitted as a series of numbers-for example, $A=65, B=66$, a space $=32$, and so on If the text is sent as 60 or 80 character chunks each character can be added up to get a sum that is the total value of that chunk. By sending that value as well as the text, the user can test whether or not he has received a corrupted version by again adding up the values and checking the sum against the transmitted value. This is called a checksum.

If the user also sends each line twice he can then, if there is a discrepancy, discard the faulty line and test the duplicate line. This method will work well provided that the error rate is not excessive. The method used here encrypts all the data including carriage returns and sends it in duplicate blocks of 80 characters that also include encrypted checksums. After the users receive the text file they run the decryption program that checks each line. As duplicate lines are sent loss of a data line is rare, but if it should happen then a line of asterisks is printed instead and a message to that effect is displayed. If it is impossible to determine the start of a line then a message is displayed requesting a retransmission of the entire file. So much for the error checking, what about the encryption?

\section{TWO POPULAR METHODS}

The two most popular methods are the data encryption standard, which was adopted in 1977 by the American Bureau of Standards, and the so called public key encryption method. The advantage of the data encryption standard technique is the large number of unique keys - that is, combination of numbers that can be used to encrypt the data-and, of course, only the identical key can be used for decryption. The total number of possible keys is $2^{56}$, which is almost $10^{17}$ ( 1 followed by 17 noughts). Our method would, at least, have to equal that level of security.

The data encryption standard is, however, meant to be implemented in hardware and that would seriously limit its application here. Similarly the public key technique is not readily applicable to microcomputers because for real security it relies on the use of numbers larger than most microcomputers can easily handle. Our method depends on altering the text by adding a random number to each character, a so called stream cipher, then putting the encrypted text into another file before that is transmitted. Again, the test of security is the number of possible keys. We can have a maximum of $10^{18}$ unique keys although it could be expanded to $10^{24}$-that is, ten million times the number provided by the data encryption standard-if it should be thought desirable.

Two small files are used to set up a pseudo random number generator. Programs are provided to generate these files, and these can be issued to approved users and renewed as frequently as thought necessary. 
All our programs are written in BBC BASIC but in a highly structured form so that they can be easily transferred to any other BASIC or any other programming language. The only machine specific features are the disc handling routines. The speed will depend on the actual implementation, but in the BBC BASIC version encryption runs at approximately 23 and decryption at approximately 28 characters a second. The size of the text files that it can handle is limited to one third of the maximum capacity of a disc. If even one character of the random generator files is wrong the output is complete nonsense. The pseudo random number generator does not repeat for more than two million characters so that large texts can be safely encrypted. The method is being used successfully in the Royal Infirmary, Edinburgh, for data transmission between the department of clinical chemistry and the medical renal unit, but a large pilot scheme is being set up with the help of the computer services unit of the South East Computer Consortium to link several other hospital laboratories and hospital departments in this way.

\section{Preliminary results}

In the case of the medical renal unit the advantage of electronic data transmission is that the decrypted data can be directly downloaded into our patient record computer. This has meant a considerable saving in secretarial time and an elimination of transcription errors. Although the medical renal unit is the greatest single user of clinical chemistry the whole process of calling up Telecom Gold, reading and decrypting the results, then loading it into our computer takes only ten minutes.

The same result could have been achieved by using a direct link over a dedicated telephone line, but this would have meant an increasing multiplicity of lines to cover links with other laboratories as they become computerised. With the present scheme neither we nor clinical chemistry need any more than a single line no matter how many laboratories or departments are connected. Calling up Telecom Gold and reading the mail does require some training, as the state of the art does not yet allow this to be completely automated. The operator must have a reasonable idea of what is going on, but the laboratory staff in either unit have not experienced any real difficulty over this. Data have been transmitted once a day five days a week since 6 May 1985 with no serious problems.
The only minor problems that we have encountered have been difficulties in calling up Telecom Gold, probably because British Telecom had underestimated the likely demand and the local $c$ number was frequently engaged. Complaints to Telecom Gold $\widehat{\widehat{\Omega}}$ have, however, led to a marked improvement and use after $11 \mathrm{am}$ seems trouble free.

As I am both the instigator and the programmer of the scheme my $\mathbb{D}$ opinion that it is working well could be considered biased. It is encouraging, however, that the users in clinical chemistry are most enthusiastic and are looking forward to the opportunity of providing data to the other departments when the pilot scheme starts.

\section{Future development}

Although the system is most useful for computer to computer data transfer, it is also true that it is much faster than the traditional $\overrightarrow{0}$ delivery van for sending data to outlying hospitals and could mean that these hospitals would receive same day service for laboratory data. The pilot scheme will include this facility. The decrypted results will probably be printed out on to facsimile forms before 3 being transferred to the wards for insertion into the patients' notes in the usual way. When the hospitals convert to computerised notes the data could again be fed directly to the computer.

Electronic mail is the natural extension of word processing so we will also be looking at the possibility of using the mail boxes for nonsensitive communications. This will become increasingly attractive as the number of Telecom Gold users increases. The pilot scheme will fully check all the above possibilities and we shall be looking both for advantages and for any unforseen problems. If the tests are successful the method will be recommended for general use in the health board's area, where staff already realise the great advantages of sending such data to general practitioners by electronic mail. The present trial is concentrating on text files only, but improved versions of the programs are available that permit the transfer of program files via Telecom Gold.

(Accepted 13 September 1985)

\section{Increased fees agreed by BMA}

Following the agreement between the BMA and the Treasury for an increase in fees regulated by the general schedule of fees for part time medical services for central government departments (20 July, p 229) agreement has now been reached on departmental schedules. These include fees for locum cover in the temporary absence of service medical officers, fees for medical boards, and fees for postmortem examinations commissioned by a pneumoconiosis panel.

Supplements for the guidance note Fees for part time medical services are being revised to reflect the new rates effective from 1 June 1985 . The supplements listed below will be available soon from BMA local offices. Members should quote their current membership number and the appropriate reference number: fees paid by DHSS (FS3A); fees paid by Ministry of Defence (FS4); fees paid by employment service division (FS5); fees paid by Department of Transport (FS6); fees paid by Home Office prison department (FS7); fees paid by Criminal Injuries Compensation Board (FS8); fees paid by Lord Chancellor's department (other than for court work) (FS9); and fees paid by Employment Medical Advisory Service (FS10).

The BMA has also concluded an agreement with the Home Office for increases in salaries of part time prison medical officers, together with increases in certain allowances paid to such medical officers. The increases take effect from 1 April. For the first hour per week for which the medical officer is committed the annual salary rises from $£ 600$ to $£ 840$. For each subsequent hour per week thereafter the annual salary is $£ 560$.

\section{Reviewing deputising arrangements}

Secretaries of local medical committees have been asked to help review arrangements introduced last year to monitor deputising services. The General Medical Services Committee has circulated a questionnaire, which it hopes will be completed by a member of the local medical committee who serves on the deputising services subcommittee of the family practitioner committee or by the secretary of the local medical committee. The committee is anxious to have as full and complete a picture as possible of how the arrangments have been received and are operating. Replies will be treated in the strictest confidence and results will be aggregated so that no individual or locality can be identified.

\section{Study shows wide variation in surgeons' workloads}

Hospitals could make better use of existing resources to reduce waiting lists, according to John Yates of the Health Services Management Centre, University of Birmingham. Writing in the November issue of NAHA News, he argued that empty beds, wasted operating theatres, and reluctant surgeons were contributing factors to the waiting list, which at present stands at over half a million patients. While accepting that there should be some spare capacity, John Yates found that: "It is an incredible paradox that the NHS should have 50000 acute beds empty each day whilst so many people are waiting for treatment." Taking ear, nose, and throat surgery as an example, he said that the average number of empty beds is $43 \%$ of that available. He also pointed to a major deficiency in the collection of data in the NHS in that for well over 30 years information about the use of operating theatres had not been systematically collected. The recent production of performance indicators by the DHSS showed the wide range of theatre sessions available in relation to the bed provision.

John Yates's research showed large differences in the workload of surgeons - for example, in some districts orthopaedic surgeons treated 100 cases a year and in others almost 1000 a year.

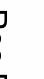

